\title{
A New Model for Characterizing Nonlinear Hysteresis of Magnetorheological Fluid Damper
}

\author{
X. J. Yuan \\ School of Automotive Engineering, Hubei University of Automotive Technology, shiyan, 442002, P.R. China. \\ Key Laboratory of Automotive Power Train and Electronics (Hubei University of Automotive Technology), Shiyan, \\ 442002, P.R. China.
}

\author{
T. Y. Tian, H. T. Ling and T. Y. Qiu \\ School of Automotive Engineering, Hubei University of Automotive Technology, Shiyan, 442002, P.R. China.
}

\begin{abstract}
(Received 1 June 2018; accepted 22 November 2018)
Magnetorheological (MR) dampers whose nonlinear hysteresis is a rather complicated phenomenon have been widespread in mechanical systems, automobile shock absorbers, the civil engineering, etc. The understanding of such a behaviour is helpful to control effectively and utilize maximum advantages of MR dampers. It is vitally important to construct parametric models used to develop control algorithms. Hence, the current study aims at developing a parametric model which exhibits considerably better predictions than that of more complicated models. In addition to achieving such a target, a simple algebraic model including only an exponential function, a hyperbolic tangent function, and other algebraic expressions can be able to capture the non-linear hysteresis adequately. Compared to an existing algebraic model and the experimental dataset, the proposed model is a reliable one.
\end{abstract}

\section{INTRODUCTION}

Serviced as damping elements for vibration control, MR dampers have been applied in automobile shock absorbers, large bridges, artillery systems, washing machines, buildings, knee prostheses and so on. ${ }^{1-4}$ Having a good understanding of nonlinear hysteresis characteristics, the effective control of these dampers can be achieved. Adequately characterizing such a nonlinear behaviour, appropriate models are extremely important to develop control algorithms and utilize maximum advantages of them. ${ }^{1,5}$ Therefore, including parameter and non-parametric ones, diverse models have been under consideration by international scholars. The following is a brief summary of relevant models.

Non-parametric models, such as Chebyshev polynomials, neural networks and fuzzy control, are able to characterize the nonlinear hysteresis effectively. ${ }^{6-10}$ However, these models are rather complicated. A large amount of experimental dataset is also required to validate. ${ }^{1}$ The parameters of non-parametric models, of course, have no clear physical meanings. ${ }^{11}$ Unlike non-parametric ones, including some mechanical elements such as linear viscous components, springs, friction and inertia, parametric models have some physical meanings. ${ }^{11} \mathrm{Com}-$ paring the experimental dataset with a model, the parameters related to mechanical components can be easily estimated. Therefore, as the most desirable ones, parametric models have been actively studied by scholars. Of those, developed by Sternway et al., the Bingham viscoplastic model is one of the earliest parameter models. ${ }^{12}$ Only including a coulomb friction element and a viscous damper, the force-displacement behaviour has been successfully captured by such a model. However, the force-velocity characteristic would not be portrayed sufficiently. ${ }^{5}$ Adopting an original Bingham model in series with a standard model of a linear solid, an extended model (visco-elastic-plastic model) has been proposed by Gamota and Filisko. ${ }^{13}$ The pre-yield hysteresis was first considered in the research of Kamath and Wereley. ${ }^{14}$ As an extension of the nonlinear biviscous model, a nonlinear hysteretic biviscous model could be used to improve such a representation and capture the force-displacement behaviour effectively. ${ }^{15}$ However, similar to the previous models, the force roll-off in the lowspeed region still couldn't be readily characterized through this extension. ${ }^{10}$

Depending on hysteretic variables, many models have been utilized to capture nonlinear hysteresis phenomenon. Of those, the Bouc-Wen model is extensively utilized to picture the hysteretic response of MR dampers. ${ }^{3,16,17}$ Adopting fewer parameters (compared with the modified one), the forcedisplacement behaviour can be captured well by a general Bouc-Wen model. ${ }^{1}$ In view of a trade-off between the complexity and accuracy, it is practically acceptable. ${ }^{17}$ However, the behaviour of a low-speed region cannot be presented effectively if the signs of the velocity and the acceleration are just opposite. ${ }^{1}$ In addition to such a problem, the undesirable singularities may occur due to the state variable. ${ }^{17}$ In this context, extending the original Bouc-Wen model, a modified model has been successfully developed by Spencer et al. ${ }^{5}$ Utilizing additional parameters in the modified model, characteristics in all regions and a higher accuracy can be obtained. ${ }^{1,5,17}$ Similar to previous models, the Bouc-Wen model and its modified ones are not associated with the frequency, amplitude and current. ${ }^{1}$ It is therefore greatly cumbersome and computationally expensive if the different combination of excitation parameters is desired. ${ }^{1,17}$ In order to overcome this defect, a generalized model related to the magnetic field has been further suggested by Spencer et al. ${ }^{5}$ Under any current, the behaviour of a MR 
damper will be better portrayed. Therefore, such a model is vitally helpful to understand the non-linear behaviour and develop control algorithms. Considering the current excitation, frequency and amplitude, a new model also depending on the general Bouc-Wen model has been suggested by Dominguez et al. ${ }^{18}$ Combining Spencer and Dominguez's contributions, this modified Bouc-Wen model can be used to efficiently predict the damping force in a design stage. However, owing to more parameters, the complexity of these modified models is unavoidably increased, which may bring great difficulties in the process of identification. ${ }^{17}$ Therefore, containing ordinary differential equations, these models are usually adopted in fields where an accurate model is required. ${ }^{17}$

Above survey would report that, although improved BoucWen models would effectively capture the behaviour and their accuracies are practically acceptable, the accuracies of the complex models are lower than those of simple algebraic models. ${ }^{1}$ It is vitally important to develop algebraic models so that the unknown parameters can be identified readily and accurately. Therefore, simple models with the excellent description of the non-linear behaviour have been actively concerned. For example, a new hysteresis model has been first suggested by Dahl. ${ }^{19}$ Based on the suggestion, a simpler and more effective model has been established by Zhou et al. ${ }^{20}$ Compared with the Bouc-Wen model, the significant advantage of these models is reflected in fewer parameters and its influence on other aspects. Utilizing a hyperbolic tangent function to predict the hysteretic behaviour, a novel model has been proposed by Kwok et al. ${ }^{17}$ Such a model is similar to an original model first suggested by Gavin et al. ${ }^{21}$ Developing by Guo and $\mathrm{Hu}$, an algebraic polynomial model is quite notable. ${ }^{22}$ Especially, they have noted that this algebraic model could capture the nonlinear hysteresis sufficiently. ${ }^{22}$ More accurate results for the non-linear behaviour will be obtained by these simple models, and such a higher accuracy cannot be achieved through the Bouc-Wen model or the modified ones. ${ }^{1}$ Only containing a simple hysteresis function and other algebraic expressions, these models are very helpful to compute efficiently in the process of parameter identification and design a controller.

Sahin et al have implemented comprehensive comparisons of some existing parametric models. ${ }^{1}$ It is clearly concluded from above discussions and the comprehensive comparisons that either the Bingham or the modified models bring difficulties in capturing the non-linear behaviour. The Bouc-Wen model and the modified ones also contain obvious defects. Notably, such as the Dahl model and its improved ones, the hyperbolic tangent hysteresis model and a simple algebraic model, these simple models are better ones currently. However, the width and the slope of a hysteresis loop in the low-speed region are difficult to portray accurately. A slowing trend of force-velocity behaviour in the high-speed region cannot be presented perfectly. Therefore, understanding and observing the nonlinear hysteresis, our team proposes another simple and novel model so that above two defects can be overcome. Introducing a simple exponential function, a hyperbolic tangent function and algebraic expressions, this model will be successfully developed. The following is the detail of the model and its validation.

\section{THE PROPOSED MODEL}

Considering the flow along an annular gap, the pressure drop, $\Delta p$, can be given by

$$
\Delta p=\frac{12 \eta L Q}{b g^{3}}+\frac{M L \tau_{q}}{g}
$$

where $b, L$ and $g$ are the average circumference, a length and the gap of an annular channel, $M$ is a coefficient associated with the velocity and its value varies between 2 and $3, \eta$ is the fluid viscosity without the applied field, $Q$ is the volumetric flow rate, and $\tau_{q}$ is the yield stress of MR fluid. ${ }^{23}$ Therefore, according to Eq. (1), the force induced by $\Delta p$ can be approximately followed that

$$
F_{f}=\Delta p A_{p}=\frac{12 \eta L Q A_{p}}{b g^{3}}+\frac{M L \tau_{q} A_{p}}{g} .
$$

In Eq. (2), $A_{p}=\frac{\pi d_{p}^{2}}{4}, b=\frac{\pi\left(d_{p}+d_{d}\right)}{2}$ and $Q=A_{p} U$ are the effective sectional area of the piston, an average circumference of the annular channel and the volumetric flow rate, $d_{p}$ and $d_{d}$ are diameters of the piston and the inner cylinder, and $U$ is the relative velocity between the piston and the cylinder. ${ }^{23}$ In addition to such a force, there is another one induced by the shear stress, which can be given by

$$
F_{s}=\tau S_{d}
$$

in which, the shear stress $\tau$ and the average lateral area $S_{d}$ can be approximated as ${ }^{24}$

$$
\begin{aligned}
\tau & =\tau_{q}+\eta \frac{d U}{d g}=\tau_{q}+\eta \frac{U}{g} \\
S_{d} & =L b .
\end{aligned}
$$

Using above equations gets

$$
F_{s}=\tau_{q} L b+\frac{\eta U L b}{g} .
$$

Depending on Eqs. (2) and (6), the total damping force produced in the annular channel of a MR damper can be given by

$$
\begin{aligned}
F_{d}=F_{f}+F_{s}= & \left(\frac{12 \eta L A_{p}^{2}}{b g^{3}}+\frac{\eta L b}{g}\right) U+ \\
& \left(\frac{M L A_{p}}{g}+L b\right) \tau_{q} \operatorname{sgn}(U) .
\end{aligned}
$$

For a linear motion damper, Eq. (7) also follows that

$$
\begin{aligned}
F_{d 1}=F_{f}+F_{s}= & \left(\frac{12 \eta L A_{p}^{2}}{b g^{3}}+\frac{\eta L b}{g}\right) \dot{x}+ \\
& \left(\frac{M L A_{p}}{g}+L b\right) \tau_{q} \operatorname{sgn}(\dot{x}) .
\end{aligned}
$$

where $\dot{x}$ is also the relative velocity given by the relative displacement $\mathrm{x}$ between the piston and the cylinder. A truth, in Eq. (8), is the absence of a shear-thinning expression, which is not in line with the actual non-linearity of the viscosity. An accurate expression for capturing the viscosity, especially in 


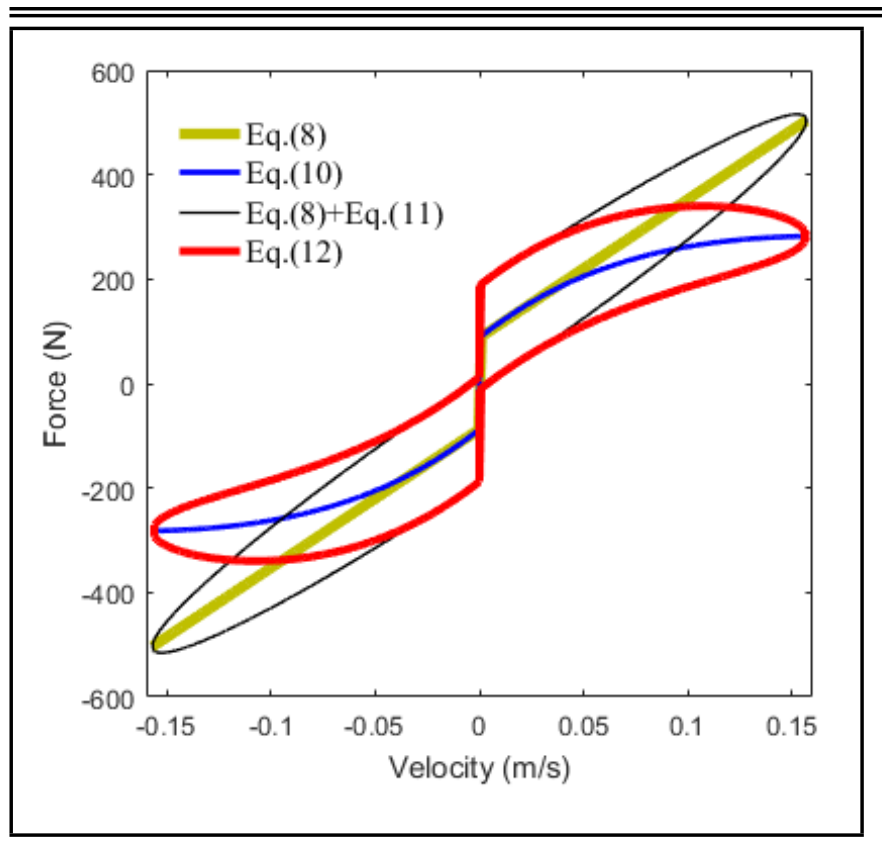

Figure 1. The function of parameters: $F_{0}=0, K=2000, C=1.2, A=6$.

the critical state between the pre-yield and post- yield regions, is extremely difficult. In addition to such a behaviour, the influence of the magnetic field on partial damping channels is different, which also makes a difference of the viscosity along the entire channel. It is therefore important to find some variables so that the accuracy of the model can be improved. In this context, a simple natural exponential function is introduced to characterize the shear-thinning phenomenon and an inconsistent behaviour. The descriptive equation is given by

$$
\eta_{d}=C \eta e^{-A|\dot{x}|}
$$

where $\eta_{d}$ is the viscosity after thinning, $A$ is a thinning factor, $C$ is a scale factor. Instead $\eta$ by $\eta_{d}$, Eq. (8) can be followed that

$$
\begin{aligned}
F_{d 2}= & \left(\frac{12 L A_{p}^{2}}{b g^{3}}+\frac{L b}{g}\right) C \eta e^{-A|\dot{x}|} \dot{x}+ \\
& \left(\frac{M L A_{p}}{g}+L b\right) \tau_{q} \operatorname{sgn}(\dot{x}) .
\end{aligned}
$$

It would appear that this damping force is only generated by the flow and the shear effect excluding other factors, such as the accumulator, friction, inertia, etc. There are, of course, two very difficult problems. One is the quantitative calculation of other forces, and other is the degree of the assisted effect on movement. Owing to these difficulties, an approximate method will be used to capture other combined effects, obtaining

$$
F_{\text {other }}=K x+F_{0} .
$$

Therefore utilizing Eqs. (10) and (11) attains

$$
\begin{aligned}
F_{d 3}= & \left(\frac{12 L A_{p}^{2}}{b g^{3}}+\frac{L b}{g}\right) C \eta e^{-A|\dot{x}|} \dot{x}+ \\
& \left(\frac{M L A_{p}}{g}+L b\right) \tau_{q} \operatorname{sgn}(\dot{x})+K x+F_{0} ;
\end{aligned}
$$

where $F_{\text {other }}$ is a force generated by other factors, $K$ is a stiffness coefficient, and $F_{0}$ is an offset. It is readily deduced from

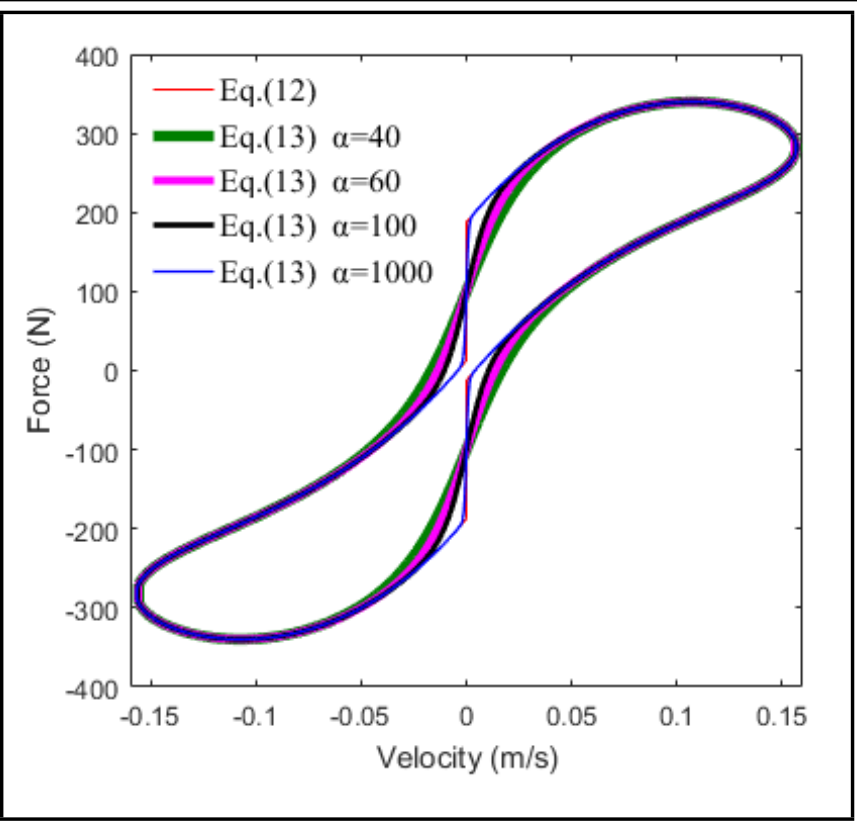

Figure 2. The function of the parameter $\alpha: F_{0}=0, K=2000, C=1.2$, $A=6$.

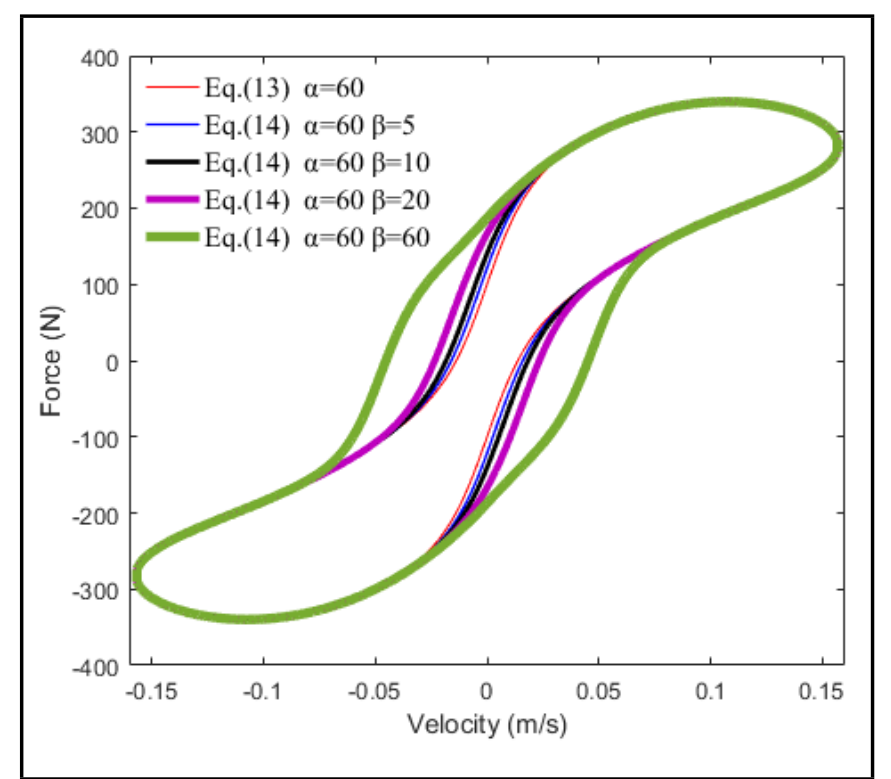

Figure 3. The function of the parameter $\beta: F_{0}=0, K=2000, C=1.2$, $A=6$.

the Fig. 1 that Eq. (12) is able to predict the rather complicated phenomenon of the nonlinear hysteresis of a MR damper, and the method combining the Bingham constitutive relationship and shear-thinning factor is useful to characterize this behaviour. However, this model wouldn't capture the characteristics in the region where the magnitude of the velocity is very small, which is known as a jump.

The simulation proves that such a behaviour does not depend on above parameters. This phenomenon is mainly caused by the sign function. It is absolutely necessary to study its influence on the low-speed region. As a result, a hyperbolic tangent function is utilized to replace the sign function. The proposed 


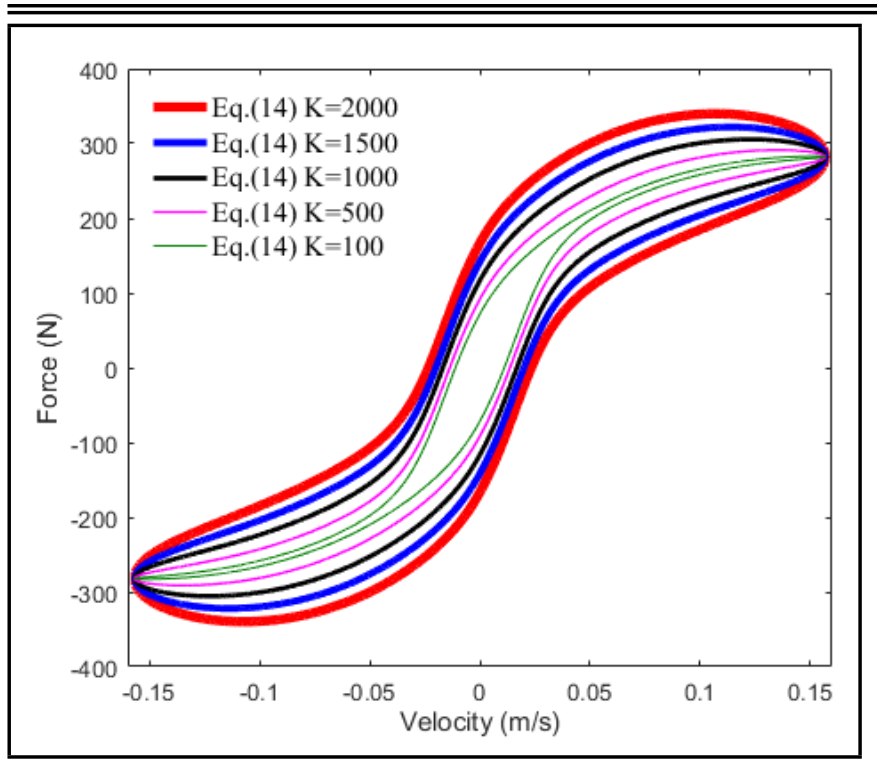

Figure 4. The function of the parameter $K: F_{0}=0, \alpha=60, \beta=20$, $C=1.2, A=6$.

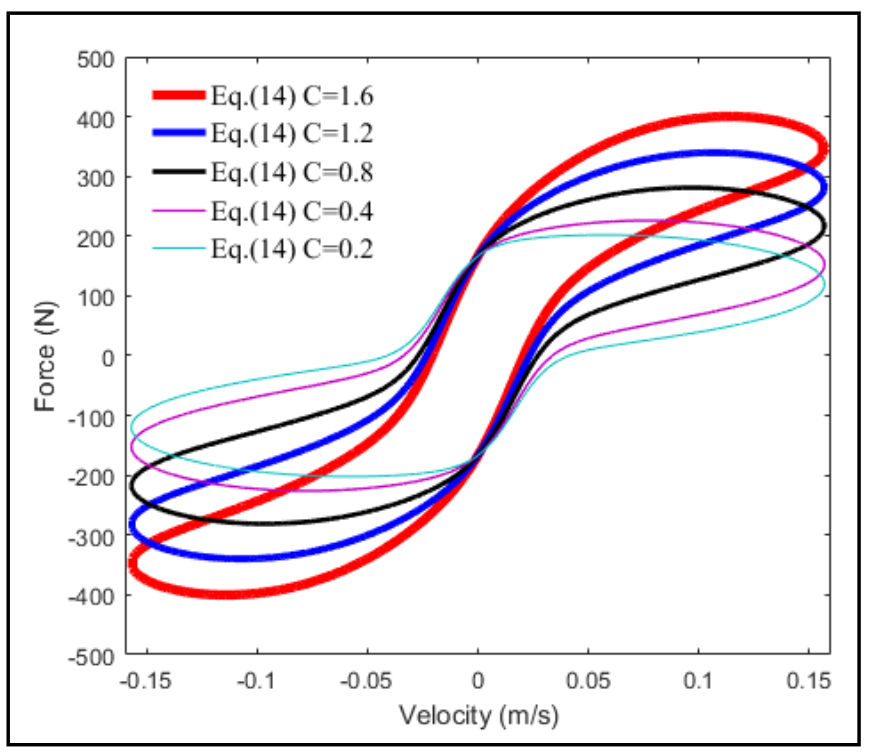

Figure 5. The function of the parameter $C: F_{0}=0, \alpha=60, \beta=20$, $A=6, K=2000$.

model can be given by

$$
\begin{aligned}
F_{d 4}= & \left(\frac{12 L A_{p}^{2}}{b g^{3}}+\frac{L b}{g}\right) C \eta e^{-A|\dot{x}|} \dot{x}+ \\
& \left(\frac{M L A_{p}}{g}+L b\right) \tau_{q} \tanh (\alpha \dot{x})+K x+F_{0} .
\end{aligned}
$$

Simulated results in Fig. 2 evidently show that almost all of curves based on Eq. (13) are in a position to capture the hysteresis and a smooth transition. A larger $\alpha$, of course, is helpful to maintain the consistency with the original model. However, similar to the sign function, the large value will lead to the jump phenomena, and a very small one will deviate from the truth. The width of the hysteresis loop is little changed in the region where the velocity is very small, and it is not able to further be adjusted by the existing parameters if $\alpha$ must be selected in this range. The appropriate $\alpha$ is also used to control the slope of the curve in the low-speed region. In this context, another displacement-correlation factor, $\beta$, is adopted to im-

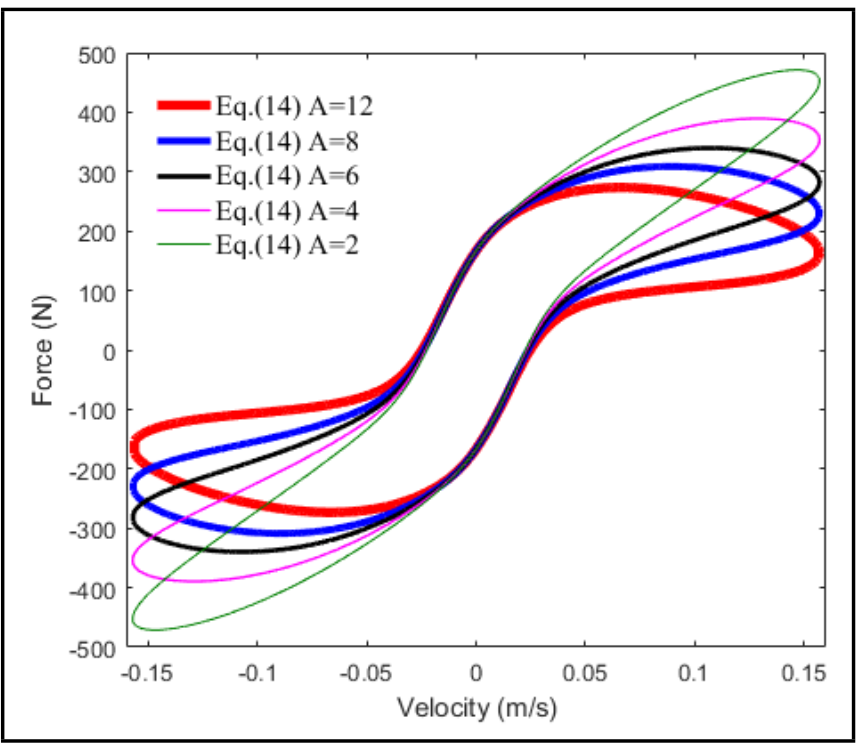

Figure 6. The function of the parameter $A$ : $F_{0}=0, \alpha=60, \beta=20$, $C=1.2, K=2000$.

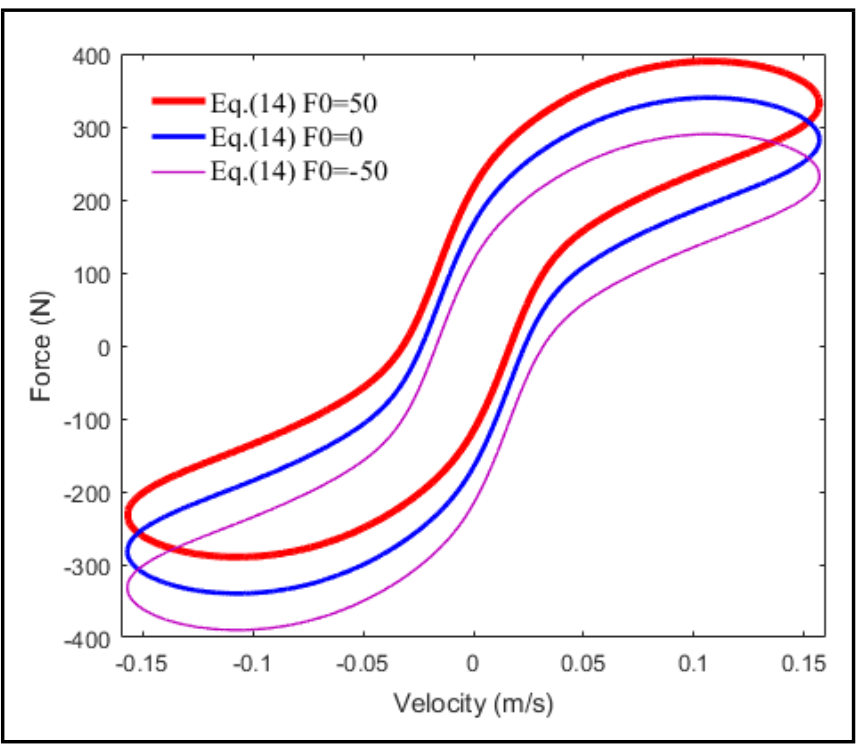

Figure 7. The function of the parameter $F_{0}: \alpha=60, \beta=20, C=1.2$, $A=6, K=2000$.

prove the width of a loop in this region. Therefore, the final model can be followed that

$$
\begin{aligned}
F_{d 4}= & \left(\frac{12 L A_{p}^{2}}{b g^{3}}+\frac{L b}{g}\right) C \eta e^{-A|\dot{x}|} \dot{x}+ \\
& \left(\frac{M L A_{p}}{g}+L b\right) \tau_{q} \tanh (\alpha \dot{x}+\beta x)+K x+F_{0} .
\end{aligned}
$$

It is readily concluded from Fig. 3 that, at a given $\alpha$, the different $\beta$ mainly influences the width of the loop in the low-speed region if it is not very large. The slope of the line in this region is governed by $\alpha$. Notably, the proper value is helpful to predict the behaviour in this region, and the predictive loop will be distorted as long as $\beta$ is too large. Therefore, during the process of identification, the value should be considered in an appropriate range.

The truth is that, as above discussion, Eq. (14) is able to characterize the non-linear behaviour in all regions, and the 


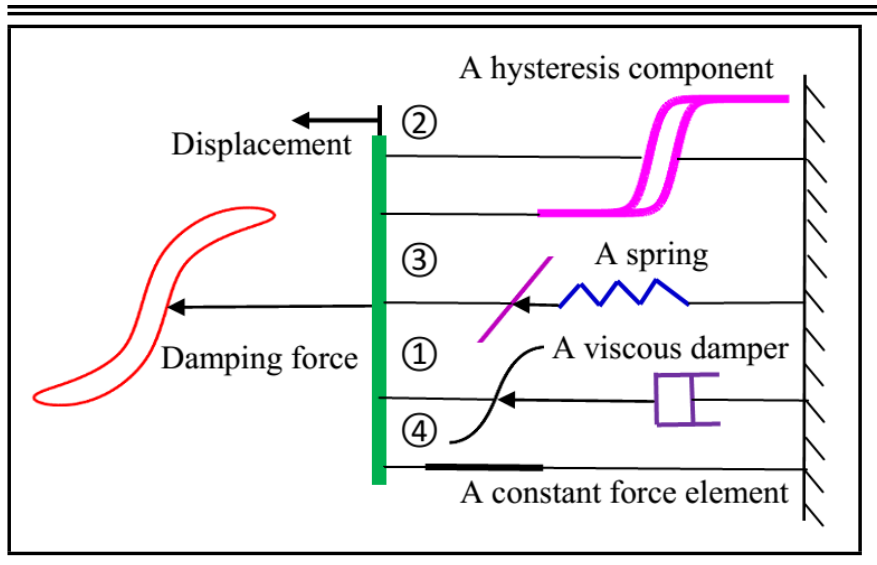

Figure 8. The configuration of the proposed model.

main functions of the parameter $\alpha$ and $\beta$ are illustrated. However, the functions of other parameters are not portrayed specifically. Therefore, mentioned in Figs. 4, 5, 6 and 7, the unique effects of them are reflected through simulation. It is readily concluded from Fig. 4 that parameter $K$ is able to control the entire width of the hysteretic loop, and the loop can be narrowed to a very small one in the high-speed region. Another truth, of course, is the limitation of reducing the loop in lowspeed region since the loop in this region will not be changed by the further decrease of $K$. This behaviour is mainly due to the effect of $\beta$ as long as the value of $K$ is reduced to some extent. It would appear that, in Fig. 5, the width of the hysteretic loop in the high-speed region cannot be enlarged or reduced. The forces will be controlled, which is consistent with the function of the scale factor $C$. As is shown in Fig. 6, the shear-thinning factor $A$ is more important to regulate the curvature of the high-speed region and adjust forces. In the proposed model, as Eq. (14) or others, the larger $A$ is able to capture the slowing trend of lines, and its appropriate value is a necessary condition for keeping consistency with the original force. The smaller $A$ is just opposite to the role of a shear-thinning factor. Notably pictured in Fig. 7, the $F_{0}$ is only utilized to move the loop parallelly.

At given parameters $\left(L=6 \mathrm{~mm}, d_{p}=40 \mathrm{~mm}, d_{d}=\right.$ $39 \mathrm{~mm}, g=0.5 \mathrm{~mm}, \eta=0.4 \mathrm{Pas}, \tau_{q}=2000 \mathrm{~Pa}, X=$ $50 \mathrm{~mm} * \sin (\pi t))$, it is easily concluded from above simulation that the proposed model is able to characterize the nonlinear hysteresis of MR dampers and each parameter has its own unique functions. However, the above conclusions are only depended on simulation and the validation is necessary. The following mainly validates the proposed model by a well-known model and the experimental dataset.

\section{VALIDATION FOR THE PROPOSED MODEL}

There is, of course, a general expression of Eq. (14). Therefore, a unitized equation is followed that

$$
\begin{aligned}
& F_{\text {damper }}= \\
& \underbrace{V S_{1} C \eta e^{-A|\dot{x}|} \dot{x}}_{\text {(1) }}+\underbrace{T(i) S_{2} \tanh (\alpha \dot{x}+\beta x)}_{\text {(2) }}+\underbrace{K x}_{\text {(3) }}+\underbrace{F_{0}}_{\text {(4) }} ;
\end{aligned}
$$

in which

$$
\begin{array}{cc}
S_{1}=\frac{12 L A_{p}^{2}}{b g^{3}}+\frac{L b}{g}, & S_{2}=\frac{M L A_{p}}{g}+L b, \\
V=\eta, & T(i)=\tau_{q} .
\end{array}
$$

It is easily found that the above model is highly associated with structural parameters and MR fluid. The coefficient $S_{1}$ and $S_{2}$ are constants. $V$ is the fluid viscosity without the applied field. $T(i)$ is the yield stress dependent on the magnetic field or an input current. Therefore, for a given structure, the relationship between the feature of MR fluid and an input current can be obtained by experiments or the theoretical calculation. Therefore, $T(i)$ will be serviced as a constant if such a relationship is established. Owing to these features of the predictive model, the unknown parameters can be easily identified by a small amount of the experimental data, which is very helpful to develop control algorithms.

Finally, depending on above simulation and the unitized equation, this model will be conceptually illustrated in Fig. 8. Containing a hysteresis component, a linear spring, a nonlinear viscous damper and a constant force element, the force range, the width of a hysteresis loop, the curvature at the high-speed region and the hysteresis slope at the low-speed region can be controlled by this model.

The above subsection mainly demonstrates the possibility of characterizing the nonlinear hysteresis in all regions. In order to validation this ability, an existing model also based on a hyperbolic tangent function will serve as a reference to examine the proposed model. In detail, the model is given by

$$
\begin{aligned}
& f=c \dot{x}+k x+\vartheta \rho+f_{0} ; \\
& \rho=\tanh (\omega \dot{x}+\varphi \operatorname{sgn}(x)) ;
\end{aligned}
$$

where $c$ and $k$ are viscous and stiffness coefficients, $\vartheta$ is a coefficient used to adjust the hysteretic loop, $\rho$ is a variable given by hyperbolic tangent function and $f_{0}$ is an offset for damping force caused by gas. ${ }^{1,17,20}$ At given currents and displacements, the relationship between parameters and input currents is determined by the literature.

Equation (17), of course, is a highly acceptable model for capturing nonlinear hysteresis. Therefore, comparisons in Fig. 9 demonstrate that this model is a reliable one. In addition to above simulation and comparisons, the test is carried out. The test equipment is indicated in Fig. 10. The parameters of a damper used in the experiment are the same as those of simulation. In each test, the excitation current of the damper is fixed, and the frequency and amplitude of the drive are also constants. Oscillation frequencies such as $0.5,1$ and $2 \mathrm{~Hz}$ can be selected, and the amplitude of the drive is limited to $25 \mathrm{~mm}$. The sampling frequency is an integer multiple of $512 \mathrm{~Hz}$. With the increase of an oscillation frequency, a larger sampling frequency should be appropriately selected. The displacement and force at each moment will be obtained. Adopting the differential method, the relationship between the force and the velocity will be further established, thus presenting the force-velocity characteristic.

Finally, the valid dataset capturing typical characteristics of a MR damper is selected to validate the model. Mentioned in 


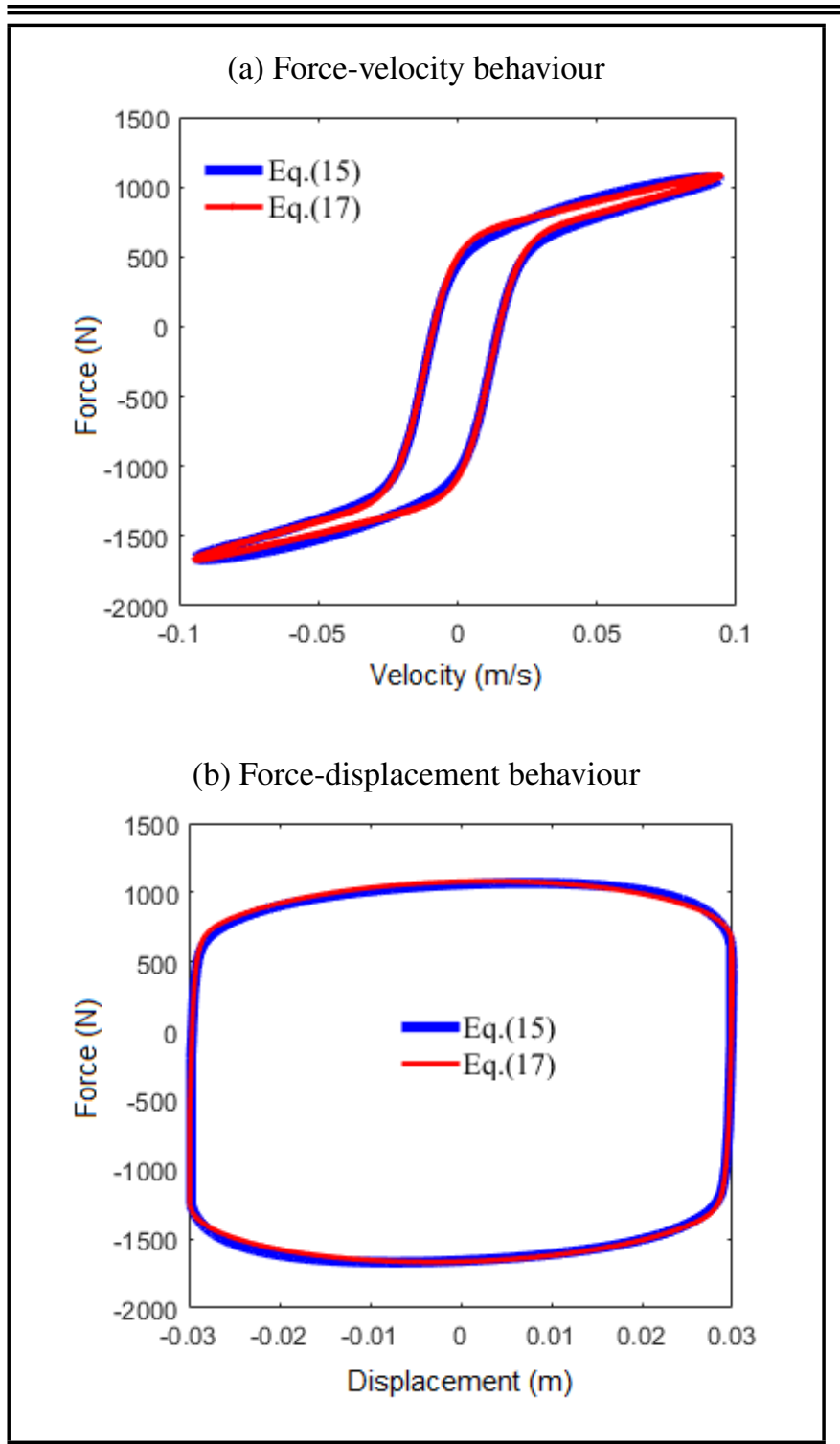

Figure 9. The comparison of two models: $i=2 \mathrm{~A}, X=0.03 \cos (\pi t)$.

Figs. 11, 12, 13 and 14, in low-speed regions, the slopes and widths of the simulated loops are highly consistent with those of experimental values. Under 0.5 and $1 \mathrm{~Hz}$ oscillation frequencies, although there are differences in transition regions, the acceptable consistencies are also reflected by comparisons in other regions. At a given oscillation frequency of $2 \mathrm{~Hz}$, the accuracy in negative regions is also quite high. Especially, the two results are basically identical if the absolute value of the speed is greater than 0.1 . However, in positive regions of highspeed, the experimental results are slightly abnormal. Abnormal behaviour is more obvious under $1.2 \mathrm{~A}$ current, which is related to defects in the design and manufacture. For a symmetric structure, behaviours are usually similar to each other in positive and negative regions. It is concluded from comparisons that features of all regions can be simulated by this model. Obvious differences in partial regions are related to the experimental data rather than defects of the model. Therefore, the proposed model is able to adequately characterize the nonlinear hysteresis of MR dampers.

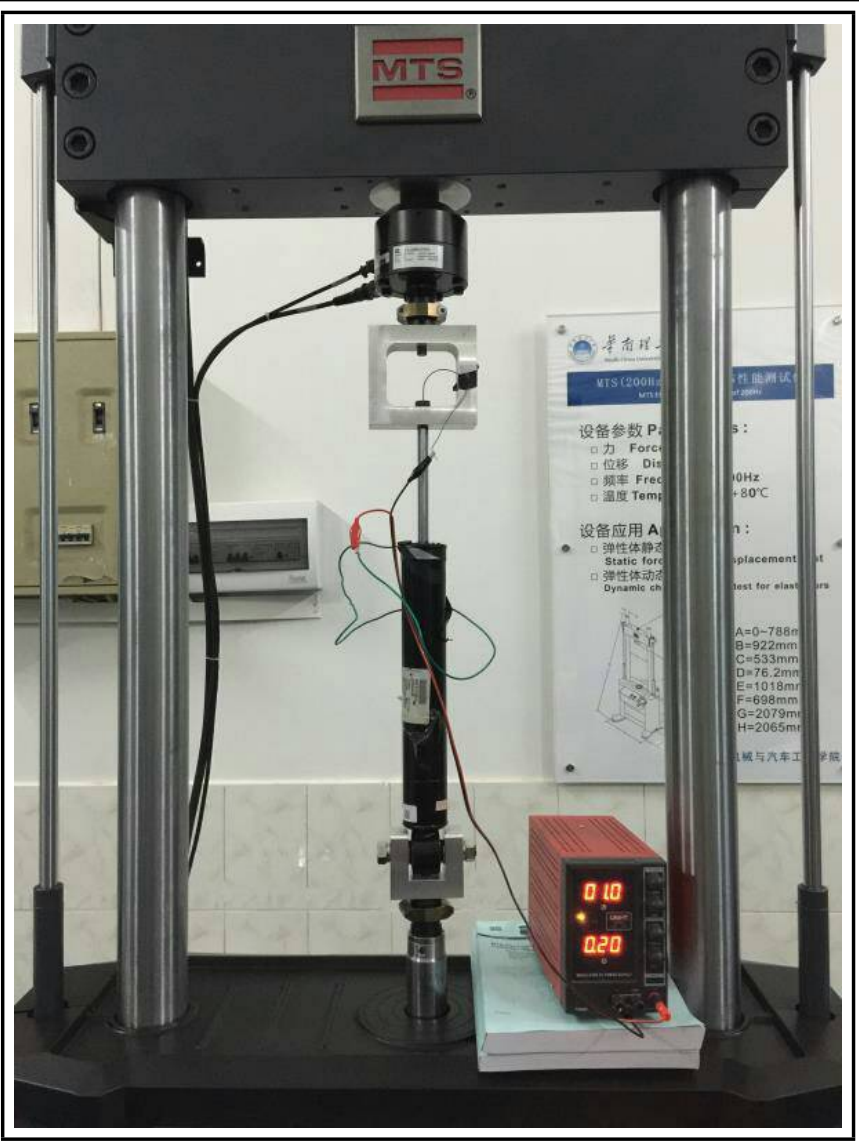

Figure 10. Test rig for a MR damper.

\section{CONLCUSIONS}

Combining the exponential function and the hyperbolic tangent function, the nonlinear behaviour of MR dampers has been successfully reflected through a very simple parameter model. It is obvious that the predictive results of this model suggested by our team are consistent with those of an existing model and the valid dataset. In order to obtain the consistency, this model is operated in such ways that

1. $\alpha$ determines the shape of the curve and mainly controls slopes of lines in the low-speed region.

2. $\beta$ is a displacement-correlation coefficient slightly adjusting width of the hysteresis loop in the low-speed region.

3. $C$ is able to control the value of the high-speed region. It doesn't influence the width of the entire hysteretic loop.

4. $A$ is the so-called thinning factor that defines a trend of damping force along with the growth of a shear rate. It is able to determine the curvature of the high-speed region and adjust forces.

5. $K$ is a stiffness coefficient greatly determining the width of the hysteretic loop, especially in high-speed region.

6. $F_{0}$ is an offset considering the inertia, friction as well as others. It is only used to parallelly move the hysteresis loop and partially reflects the force asymmetry in the compression and rebound stages. 


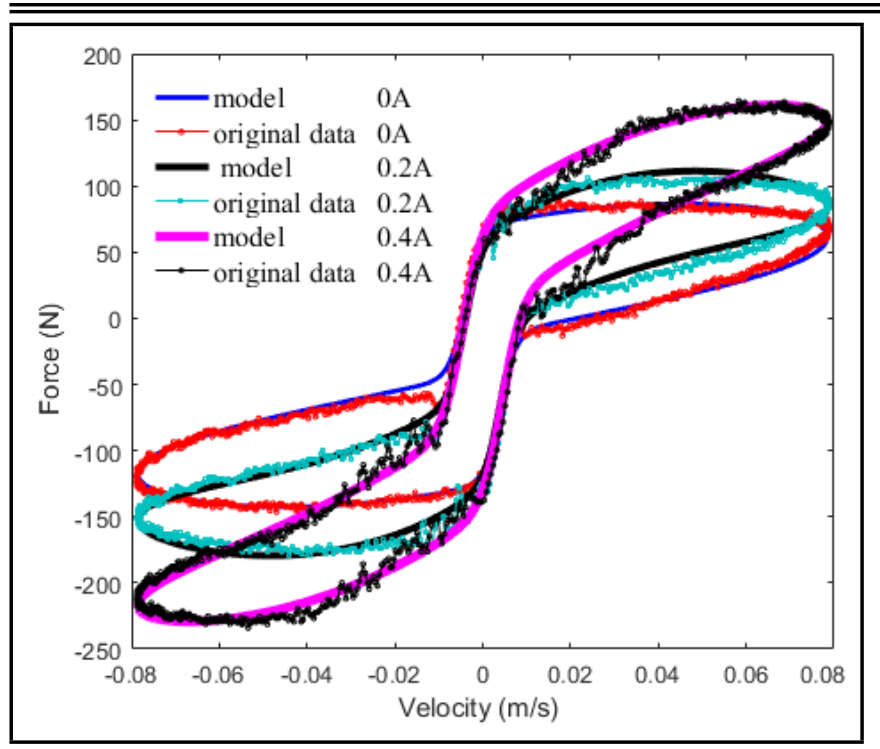

Figure 11. The predictive results and experimental dataset for $0.5 \mathrm{~Hz}$ oscillation frequency with $25 \mathrm{~mm}$ displacement amplitude at 0, 0.2, 0.4 A current.

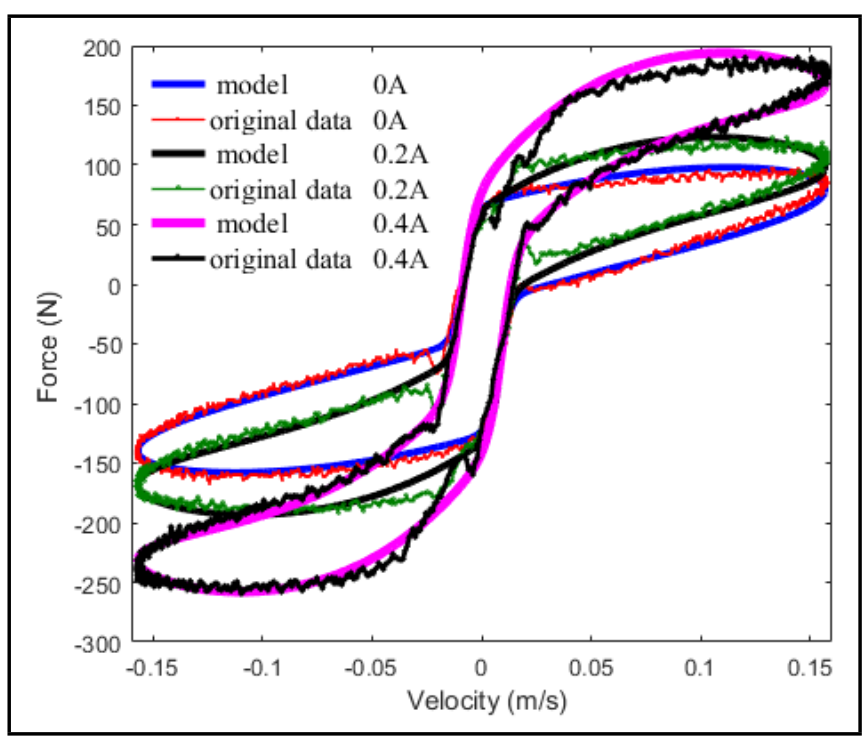

Figure 12. The predictive results and experimental dataset for $1 \mathrm{~Hz}$ oscillation frequency with $25 \mathrm{~mm}$ displacement amplitude at $0,0.2,0.4$ A current.

7. The yield stress, $\tau_{q}$, can be expressed as a function of the current as long as the structure, materials and MR fluid properties are determined, and this function also varies with the structure and MR fluid. The displacement and velocity also contain frequency information.

8. The units of $\alpha, \beta, C, A, K$ and $F_{0}$ are respectively served as $\mathrm{s} / \mathrm{m}, 1 / \mathrm{m}, 1, \mathrm{~s} / \mathrm{m}, \mathrm{N} / \mathrm{m}$ and $\mathrm{N}$.

Owing to the unique function in capturing the force-velocity characteristics in all regions, the predicted values are highly consistent with an existing model. The high consistency is also reflected through the comparisons to negative damping forces of a damper. The positive damping forces, especially under the high frequency and large currents, are slightly abnormal. Abnormal behaviour is mainly caused by manufacturing accuracy. However, the trend of force-velocity characteristics still be predicted well. Therefore, the proposed model is a relatively accurate one and presents great flexibility in simulating damping forces of MR dampers.

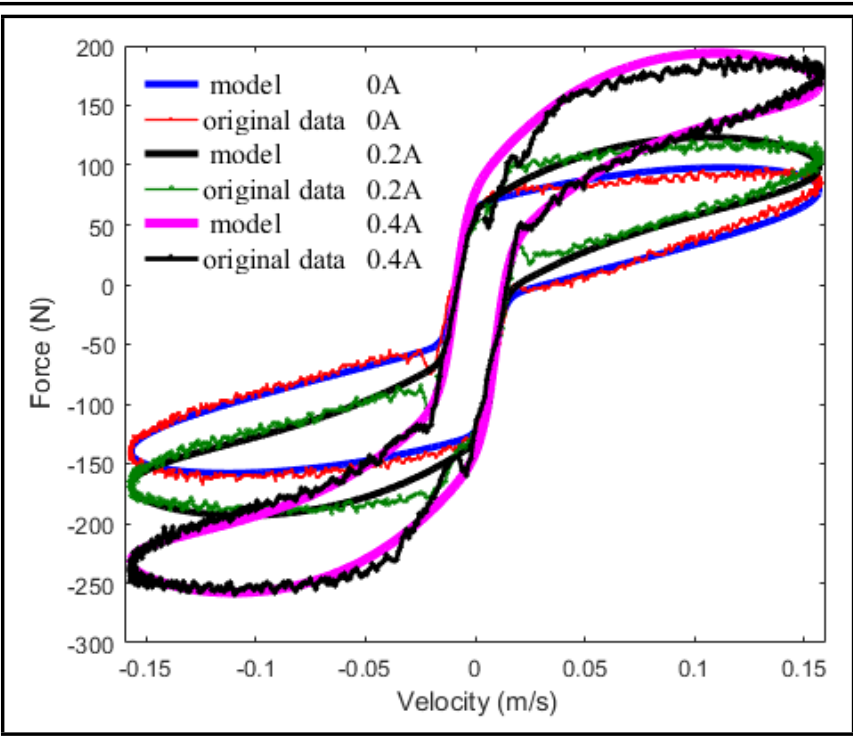

Figure 13. The predictive results and experimental dataset for $2 \mathrm{~Hz}$ oscillation frequency with $25 \mathrm{~mm}$ displacement amplitude at 0, 0.2, 0.4 A current.

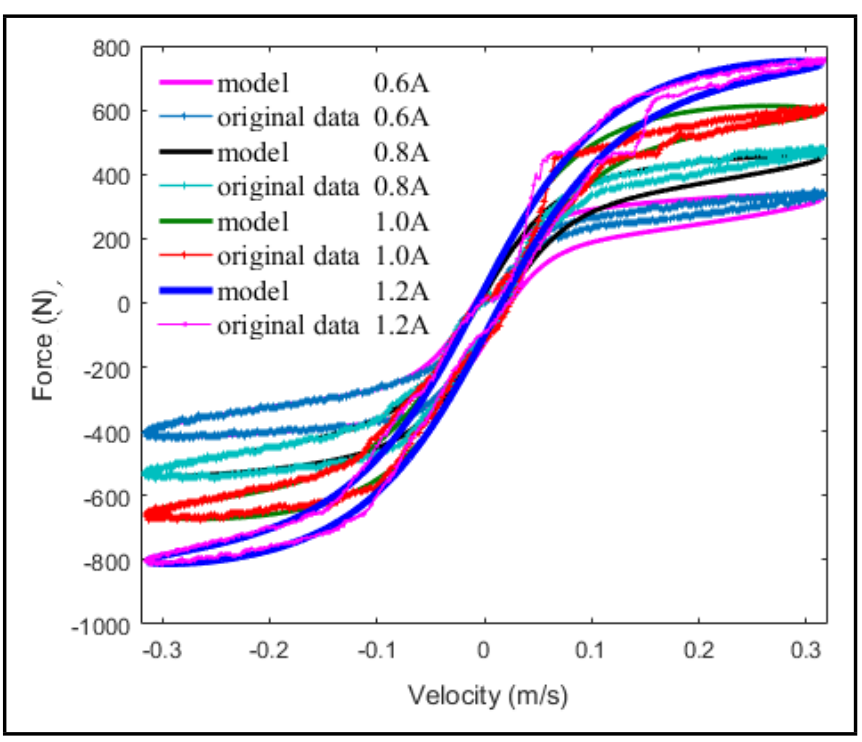

Figure 14. The predictive results and experimental dataset for $2 \mathrm{~Hz}$ oscillation frequency with $25 \mathrm{~mm}$ displacement amplitude at $0.6 \mathrm{~A}, 0.8 \mathrm{~A}, 1.0 \mathrm{~A}, 1.2 \mathrm{~A}$ current.

\section{ACKNOWLEDGEMENT}

The authors gratefully acknowledge that the work described here was financially supported by the Open Fund Project (No.ZDK1201604) sponsored by Key Laboratory of Automotive Power Train and Electronics (Hubei University of Automotive Technology) and Innovation Fund Project (No.2015XTZX0402) funded by Hubei Collaborative Innovation Center for Automotive Components Technology as well as Key Laboratory of Automotive Power Train and Electronics (Hubei University of Automotive Technology). The first author also sincerely thanks South China University of Technology for allowing experiments to be made in a test rig of MTS 370.

\section{REFERENCES}

1 Şahin, İ., Engin, T., and Şeşmeci, Ş. Comparison of some existing parametric models for magnetorheological fluid 
dampers, Smart Materials and Structures, 19 (3), 035012, (2010). https://dx.doi.org/10.1088/0964-1726/19/3/035012

2 Crivellaro, C. and Donha, D. C. Discrete-time dynamic model of a magneto-rheological damper for semi-active control design, ABCM Symposium Series in Mechatronics, 3, 27-36, (2008). Retrieved from pdfs.semanticscholar.org/7618/4b9ebe823c9f0a4f7f1e083c 40af77b496a8.pdf

${ }^{3}$ Smith K. A. Physics-based modeling of Magneto Rheological Dampers, Ph.D. thesis, University of Colorado at Boulder, USA, (2008). Retrieved from search.proquest.com/docview/304640736

${ }^{4}$ Aydar, G. A new magneto-rheological fluid (MRF) washing machine damper and a novel two-waycontrollable MRF valve, Ph.D. thesis, University of Nevada, Reno, USA (2007). Retrieved from search.proquest.com/docview/304843188

5 Spencer, B. F., Dyke, S. J., Sain, M. K., and Carlson, J. Phenomenological model for magnetorheological dampers, Journal of Engineering Mechanics, 123 (3), 230-238, (1997). https://dx.doi.org/10.1061/(ASCE)0733-9399

${ }^{6}$ Ehrgott, R. C. and Masri, S. F. Modeling the oscillatory dynamic behaviour of electrorheological materials in shear, Smart Materials and Structures, 1 (4), 275, (1992). https://dx.doi.org/10.1088/0964-1726

${ }^{7}$ Gavin, H. P., Hanson, R. D., and Filisko, F. E. Electrorheological dampers, part II: testing and modeling, Journal of Applied Mechanics, 63 (3), 676-682, (1996). https://dx.doi.org/10.1115/1.2823349

8 Wang, D. H. and Liao, W. H. Modeling and control of magnetorheological fluid dampers using neural networks, Smart Materials and Structures, 14 (1), 111, (2004). https://dx.doi.org/10.1088/0964-1726/14/1/011

9 Du, H., Lam, J., and Zhang, N. Modelling of a magneto-rheological damper by evolving radial basis function networks, Engineering Applications of Artificial Intelligence, 19 (8), 869-881, (2006). https://dx.doi.org/10.1016/j.engappai.2006.02.005

10 Wilson, C. M. D. and Abdullah, M. M. Structural vibration reduction using fuzzy control of magnetorheological dampers, Proceedings of the Structures Congress 2005: Metropolis and Beyond, 1-12, (2005). https://dx.doi.org/10.1061/40753(171)198

11 Boada, M. J. L., Calvo, J. A., Boada, B. L., and Diaz, V. Modeling of a magnetorheological damper by recursive lazy learning, International Journal of Non-Linear Mechanics, 46 (3), 479-485, (2011). https://dx.doi.org/10.1016/j.ijnonlinmec.2008.11.019

${ }^{12}$ Stanway, R. S. J. L., Sproston, J. L., and Stevens, N. G. Non-linear modelling of an electro-rheological vibration damper, Journal of Electrostatics, 20 (2), 167-184, (1987). https://dx.doi.org/10.1016/0304-3886(87)90056-8
${ }^{13}$ Gamota, D. R. and Filisko, F. E. Dynamic mechanical studies of electrorheological materials: moderate frequencies, Journal of Rheology, 35 (3), 399-425, (1991). https://dx.doi.org/10.1122/1.550221

${ }^{14}$ Kamath, G. M. and Wereley, N. M. A nonlinear viscoelastic-plastic model for electrorheological fluids, Smart Materials and Structures, 6 (3), 351, (1997). https://dx.doi.org/10.1088/0964-1726/6/3/012

15 Wereley, N. M., Pang, L., and Kamath, G. M. Idealized hysteresis modeling of electrorheological and magnetorheological dampers, Journal of Intelligent Material Systems and Structures, 9 (8), 642-649, (1998). https://dx.doi.org/10.1177/1045389X9800900810

16 Wen, Y. K. Method for random vibration of hysteretic systems, Journal of the Engineering Mechanics Division, 102 (2), 249-263, (1976).

17 Kwok, N. M., Ha, Q. P., Nguyen, T. H., Li, J., and Samali, B. A novel hysteretic model for magnetorheological fluid dampers and parameter identification using particle swarm optimization, Sensors and Actuators A: Physical, 132 (2), 441-451, (2006). https://dx.doi.org/10.1016/j.sna.2006.03.015

18 Dominguez, A., Sedaghati, R., and Stiharu, I. A new dynamic hysteresis model for magnetorheological dampers, Smart Materials and Structures, 15 (5), 1179, (2006). https://dx.doi.org/10.1088/0964-1726/15/5/004

19 Dahl, P. R. Solid friction damping of mechanical vibrations, AIAA Journal, 14 (12), 1675-1682, (1976). https://dx.doi.org/10.2514/3.61511

${ }^{20}$ Zhou, Q., Nielsen, S. R., and Qu, W. L. Semiactive control of three-dimensional vibrations of an inclined sag cable with magnetorheological dampers, Journal of Sound and Vibration, 296 (1), 1-22, (2006). https://dx.doi.org/10.1016/j.jsv.2005.10.028

${ }^{21}$ Gavin, H., Hoagg, J., and Dobossy, M. Optimal design of MR dampers, Proceedings of the US-Japan Workshop on Smart Structures for Improved Seismic Performance in Urban Regions, 14, 225-236, (2001). Retrieved from people.duke.edu/ hpgavin/ermr/seattle01b.pdf

${ }^{22}$ Guo, D. and Hu, H. Nonlinear stiffness of a magnetorheological damper, Nonlinear Dynamics, 40 (3), 241-249, (2005). https://dx.doi.org/10.1007/s11071-005-6464-y

${ }^{23}$ Wang, D. H. and Wang, T. Principle, design and modeling of an integrated relative displacement self-sensing magnetorheological damper based on electromagnetic induction, Smart Materials and Structures, 18 (9), 095025, (2009). https://dx.doi.org/10.1088/0964-1726/18/9/095025

${ }^{24}$ Wereley, N. M., Cho, J. U., Choi, Y. T., and Choi, S. B. Magnetorheological dampers in shear mode, Smart Materials and Structures, 17 (1), 015022, (2007). https://dx.doi.org/10.1088/0964-1726/17/01/015022 